\title{
Optimal design of an Otto cycle based on thermal criteria
}

\author{
Mohammad H. Ahmadi ${ }^{1, a}$, Mohammad-Ali Ahmadi ${ }^{2}$, Mehdi Mehrpooya $^{1}$, Michel Feidt $^{3}$ \\ AND Marc A. Rosen ${ }^{4}$ \\ 1 Department of Renewable Energies, Faculty of New Science and Technologies, University of Tehran, Tehran, Iran \\ 2 Department of Petroleum Engineering, Ahwaz Faculty of Petroleum Engineering, Petroleum University of Technology (PUT), \\ Ahwaz, Iran \\ 3 Laboratoire d'Energétique et de Mécanique Théorique et Appliquée, ENSEM, 2 avenue de la Forêt de Haye 60604, \\ 54518 Vandoeuvre, France \\ 4 Faculty of Engineering and Applied Science, University of Ontario Institute of Technology, 2000 Simcoe Street North, Oshawa, \\ Ontario, L1H 7K4, Canada
}

Received 23 December 2014, Accepted 19 June 2015

\begin{abstract}
In recent years, numerous analyses have been performed on Otto cycles and Otto engines, but these have often yielded different output powers and engine thermal efficiencies. In the present study, output power and engine thermal efficiency are optimized and entropy generation is minimized using a NSGA algorithm and thermodynamic analysis. The Pareto optimal frontier is obtained and a final optimal solution is selected using various decision-making approaches, including fuzzy Bellman-Zadeh, LINMAP and TOPSIS methods. The results enhance understanding of the performances of Otto cycles and of their optimization.
\end{abstract}

Key words: Otto cycle / thermodynamic analysis / power / NSGA / thermal efficiency / entropy

\section{Introduction}

Recently, finite-time thermodynamics has helped to improve various optimization objectives in the analysis and optimization of thermodynamic cycles [1-10]. Optimal performance when specific heats are constant and when they are variable, or when their ratio is variable, has been the focus of several Otto cycle investigations. Klein [11] studied the dependence of the work output on compression ratio of an endoreversible Otto cycle. Wu and Blank [12] investigated the effect of combustion on the performance of endoreversible Otto cycle. They also examined the optimization of the output power and the pressure of the Otto cycle [13].

Finite-time thermodynamic analysis was employed to derive of the output work and the efficiency relationship of the endoreversible air-standard Otto cycle by Chen et al. [14]. Ficher and Hoffman [15] investigated how a Novikov model for an endoreversible Otto cycle could provide the basis for a quantitative simulation with reasonable accuracy. Ozsoysal [16] studied the heat loss characters of an endoreversible Otto cycle. Hou [17] reviewed methods for the evaluation of the output work and efficiency of endoreversible Otto and Atkinson cycles, and

\footnotetext{
a Corresponding author: mehrpoya@ut.ac.ir
}

demonstrated that the Atkinson cycle has a greater work output under the same conditions.

Angulo-Brown et al. [18, 19] modeled an irreversible Otto cycle with friction loss over a limited time. In a different investigation, Angulo-Brown et al. [20] employed the ratio of various entropy changes to determine internal irreversibilities. Chen et al. [21] employed compression and expansion efficiencies to identify the internal irreversibility. Zhao and Chen [22] used the irreversibility model of Chen et al. [21] to investigate the output power and the efficiency of an irreversible Otto cycle.

Feidt [23] employed the maximum output work to optimize the end temperature of the compression stroke of an irreversible Otto cycle. Rocha-Matinez et al. [24] modeled an irreversible Otto-cycle.

Multi-objective optimization is increasingly used in engineering problems, e.g., skyline computation and vehicle routing [25-27]. The solution of multi-objective optimization problems requires that a number of different and sometimes conflicting objectives is simultaneously satisfied. Evolutionary algorithms (EAs) were developed and applied during the 20th century in an effort to stochastically solve problems of this generic class [28]. A reasonable solution to a multi-objective problem is a balance among a set of solutions, where each of which satisfies the 


\section{Nomenclature}

\begin{tabular}{|ll|}
\hline$A$ & Rate of heat release by fuel $(\mathrm{kW})$ \\
$B$ & Constant related to heat transfer $\left(\mathrm{kJ} \cdot \mathrm{kg}^{-1} \cdot \mathrm{K}^{-1}\right)$ \\
$C$ & Specific heat $\left(\mathrm{kJ} \cdot \mathrm{kg}^{-1} \cdot \mathrm{K}^{-1}\right)$ \\
$d$ & Ecological function $(\mathrm{kW})$ \\
$f$ & Deviation index \\
$k$ & Friction force $\left(\mathrm{kg} \cdot \mathrm{m} \cdot \mathrm{s}^{-2}\right)$ \\
$L$ & Adiabatic exponent \\
$M$ & Stroke length $(\mathrm{m})$ \\
$n$ & Cycles running per second $(\mathrm{kJ})$ \\
$P$ & Output power $(\mathrm{kW})$ \\
$Q$ & Heat transfer rate added or rejected by the working fluid $(\mathrm{kW})$ \\
$T$ & Temperature $(\mathrm{K})$ \\
$V$ & Volume $\left(\mathrm{m}^{3}\right)$ \\
$\nu$ & Velocity $\left(\mathrm{m} \cdot \mathrm{s}^{-1}\right)$ \\
$X$ & Vector of decision variables \\
$X_{1}$ & Piston positions at maximum volume \\
$X_{2}$ & Piston positions at minimum volume \\
\hline & $\quad$ Greek letters \\
\hline$\lambda$ & Compression ratio \\
$\eta$ & Thermal efficiency \\
$\eta_{c}$ & Compression efficiency \\
$\eta_{e}$ & Expansion efficiency \\
$\mu$ & Fuzzy membership function \\
$\mu^{\prime}$ & Friction coefficient $\left(\mathrm{kg} \cdot \mathrm{s}^{-1}\right)$ \\
$\sigma$ & Entropy generation rate $(\mathrm{kW})$ \\
\hline & \\
\hline$E$ & Maximum ecological function point \\
$i n$ & Heat added \\
$l e a k$ & Heat leakage \\
$o t$ & Otto cycle \\
$p q$ & Exhaust stroke \\
$q$ & Effect of heat transfer \\
$\mu^{\prime}$ & Effect of friction loss \\
0 & Environment \\
\hline-4 & State point \\
\hline
\end{tabular}

objectives at a plausible level but is not dominated by any other solution [29]. Multi-objective optimization problems generate an unlimited collection of solutions, the Pareto frontier, whose vectors illustrate the best feasible tradeoffs in the objective function space. Multi-objective optimizations of various themodynamic and energy systems have been performed recently [30-37].

To account for all of the above issues, we consider three objective functions here: output power, thermal efficiency of the overall Otto engine and entropy generation. In addition, the multi-objective optimization is conducted with three decision variables: the friction coefficient, a constant related to heat transfer and the compression ratio. Specifically, output power and thermal efficiency are maximized and entropy generation minimized via the multi-objective optimization algorithms implemented in this study. Also, error analyses were conducted to ascer- tain the robustness and precision of the solutions obtained with various decision-making approaches.

\section{Thermodynamic model}

The practical four-stroke internal combustion engine cycle consists of four main processes: intake, compression, expansion and exhaust. The practical internal combustion cycle is open. Using the air standard assumption, the open practical cycle can be presented as closed ideal cycle with the air as the working fluid. An air standard Otto cycle model is shown in Figure 1 . Process $1 \rightarrow 2 S$ represents reversible adiabatic (isentropic) compression, while process $1 \rightarrow 2$ is an irreversible adiabatic process that takes into account the internal irreversibility in the real compression process. Similarly, process $3 \rightarrow 4 S$ represents 


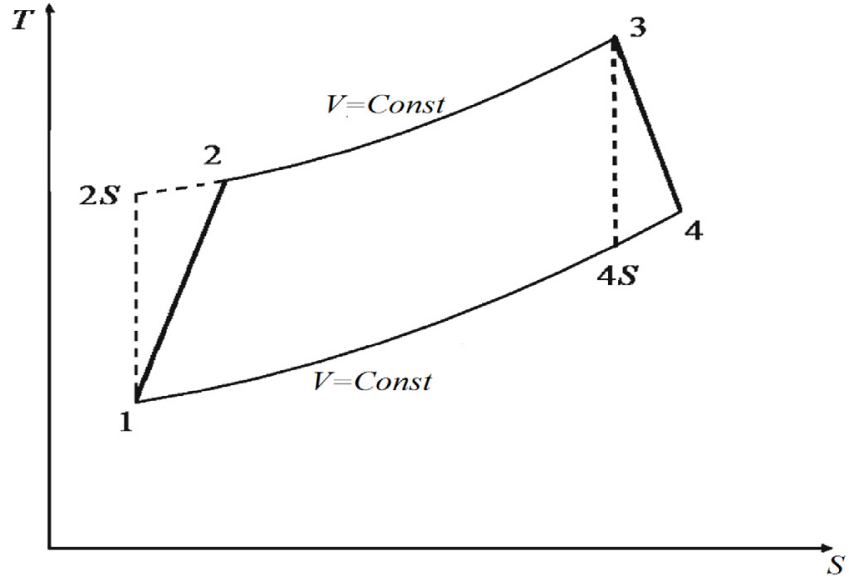

Fig. 1. T-S diagram for an irreversible Otto cycle.

reversible adiabatic expansion, and process $3 \rightarrow 4$ irreversible adiabatic expansion, with the latter accounting for internal irreversibilities in the real process. Process $2 \rightarrow 3$ represents isochoric heat addition, and process $4 \rightarrow 1$ isochoric heat rejection.

The heat addition rate to the working fluid during process $2 \rightarrow 3$ can be written as

$$
Q_{i n}=M C_{v}\left(T_{3}-T_{2}\right)
$$

where $C_{v}$ is the specific heat at constant volume of the working fluid, which is unchanged during the cycle, and $M$ is the mass flow rate of the working fluid. Similarly, the heat rejection rate by the working fluid during process $4 \rightarrow 1$ is

$$
Q_{\text {out }}=M C_{v}\left(T_{4}-T_{1}\right)
$$

The compression ratio $\lambda$ is defined as

$$
\lambda=\frac{V_{1}}{V_{2}}
$$

Therefore, the following relations can be written for reversible adiabatic processes $1 \rightarrow 2 S$ and $3 \rightarrow 4 S$ [38]:

$$
\begin{aligned}
& T_{2 s}=T_{1} \lambda^{k-1} \\
& T_{4 s}=T_{3} \lambda^{1-k}
\end{aligned}
$$

where $k$ is the adiabatic exponent.

For the two adiabatic processes $1 \rightarrow 2$ and $3 \rightarrow 4$, the compression and expansion efficiencies can be defined as [38]:

$$
\begin{aligned}
& \eta_{c}=\frac{\left(T_{2 s}-T_{1}\right)}{\left(T_{2}-T_{1}\right)} \\
& \eta_{e}=\frac{\left(T_{4}-T_{3}\right)}{\left(T_{4 s}-T_{3}\right)}
\end{aligned}
$$

These two efficiencies can be used to describe all the internal irreversibility losses, which include friction losses in irreversible compression and expansion. Substituting
Equations (4) and (5) into Equations (6) and (7), respectively, yields [38]

$$
\begin{aligned}
& T_{2}=\frac{T_{1}\left(\lambda^{k-1}-1\right)}{\eta_{c}}+T_{1} \\
& T_{4}=\eta_{e} T_{3}\left(\lambda^{1-k}-1\right)+T_{3}
\end{aligned}
$$

An ideal Otto cycle has no heat losses. In a real Otto cycle, however, there is heat transfer irreversibility between the working fluid and the cylinder wall. Heat loss through the cylinder wall is dependent on the average temperatures of the working fluid and the cylinder wall. The wall temperature is constant at $T_{0}$. If the heat rate released by combustion is $A_{1}$ and the heat leakage coefficient for the cylinder wall is $B_{1}$, the combustion heat rate added to the working fluid can be written as a linear equation as follows $[11,14,39]$ :

$$
Q_{i n}=A_{1}-M B_{1}\left[\frac{\left(T_{2}+T_{3}\right)}{2}-T_{0}\right]
$$

From Equation (10), one can see that the heat rate added to the cycle consists of two parts: the heat rate released by combustion and the heat leakage rate, which can be written as [38]

$$
Q_{\text {leak }}=M B\left[T_{2}+T_{3}-2 T_{0}\right]
$$

Here, $B=B_{1} / 2$ is a constant associated with heat transfer. There exists friction loss during the movement of the piston, and the internal irreversibility loss in compression and expansion is mainly caused by friction losses in these two strokes. The friction coefficient on the intake stroke is reported to be three times greater than the friction coefficient on the exhaust, mainly due to the pressure drop loss [40-42]. For the dual cycle, the following expression can be applied $f_{\mu^{\prime}}=-\mu^{\prime} v=-\mu^{\prime} \mathrm{d} X / \mathrm{d} t$, where $X$ is the piston displacement. Hence, the power loss caused by friction loss during the intake and exhaust strokes of overall cycle can be expressed as $[38,42]$

$$
P_{\mu^{\prime}}=\frac{\mathrm{d} W_{\mu^{\prime}}}{\mathrm{d} t}=4 \mu^{\prime} \frac{\mathrm{d} X}{\mathrm{~d} t} \frac{\mathrm{d} X}{\mathrm{~d} t}=4 \mu^{\prime} \nu^{2}
$$

If we consider a four stroke engine, the total distance that the piston travels per cycle is $4 L$ [38], where

$$
4 L=4\left(X_{1}-X_{2}\right)
$$

Here, $X_{1}$ and $X_{2}$ are the piston positions at maximum and minimum volume, respectively, and $L$ is the stroke length.

For a four stroke cycle engine, operating at $n$ cycles per second, the mean velocity of the piston is [38]

$$
\bar{\nu}=4 L n
$$

Then, the lost power due to friction loss is [38]

$$
P_{\mu}=4 \mu^{\prime}(4 L n)^{2}
$$


Thus, the power output of the cycle is [38]

$$
P_{o t}=M C_{v}\left(T_{3}+T_{1}-T_{2}-T_{4}\right)-64 \mu^{\prime}(L n)^{2}
$$

and the thermal efficiency of the cycle is [38]

$$
\eta_{o t}=\frac{P_{o t}}{Q_{\text {in }}+Q_{\text {leak }}}
$$

In a real Otto cycle, there are friction, heat transfer and internal irreversible losses. The entropy generation rate due to heat transfer and friction losses, respectively, can be written as [38]

$$
\begin{aligned}
\sigma_{q} & =\frac{Q_{\text {leak }}}{T_{0}}=\frac{M B\left(T_{2}+T_{3}-2 T_{0}\right)}{T_{0}} \\
\sigma_{\mu^{\prime}} & =\frac{P_{\mu^{\prime}}}{T_{0}}=\frac{64 \mu^{\prime}(L n)^{2}}{T_{0}}
\end{aligned}
$$

The entropy generation rate due to the loss of irreversible compression and expansion can be calculated by the entropy increase rate of processes $2 S \rightarrow 2$ and $4 S \rightarrow 4$, respectively, as follows [38]:

$$
\begin{aligned}
& \sigma_{2 S \rightarrow 2}=M C_{v} \ln \left(\frac{T_{2}}{T_{2 S}}\right) \\
& \sigma_{4 S \rightarrow 4}=M C_{v} \ln \left(\frac{T_{4}}{T_{4 S}}\right)
\end{aligned}
$$

The working fluid is discharged to the atmosphere. The entropy generation rate caused by the exhaust stroke can be calculated as follows [38]:

$$
\sigma_{p q}=M C_{v}\left[\frac{\left(T_{4}-T_{1}\right)}{T_{0}}-\ln \left(\frac{T_{4}}{T_{1}}\right)\right]
$$

Thus, the entropy generation rate of the overall cycle is $[38]$

$$
\sigma_{o t}=\sigma_{q}+\sigma_{\mu^{\prime}}+\sigma_{2 S \rightarrow 2}+\sigma_{4 S \rightarrow 4}+\sigma_{p q}
$$

Following the definition of the ecological function in reference [43], the ecological function of the irreversible Otto cycle is

$$
E_{o t}=P_{o t}-T_{0} \sigma_{o t}
$$

\section{Multi-objective optimization with evolutionary algorithms}

\subsection{Optimization via EAs}

John Holland developed the genetic algorithm (GA) in the $1960 \mathrm{~s}$ in order to import the mechanisms of natural adaptation into computer algorithms and numerical optimization [25]. Genetic algorithms apply an iterative, stochastic search strategy to find the best answer and mimic in a straightforward way the principles of biological evolution (Fig. 2). In this paper, a genetic algorithm is used to obtain the Pareto frontier. This method is a

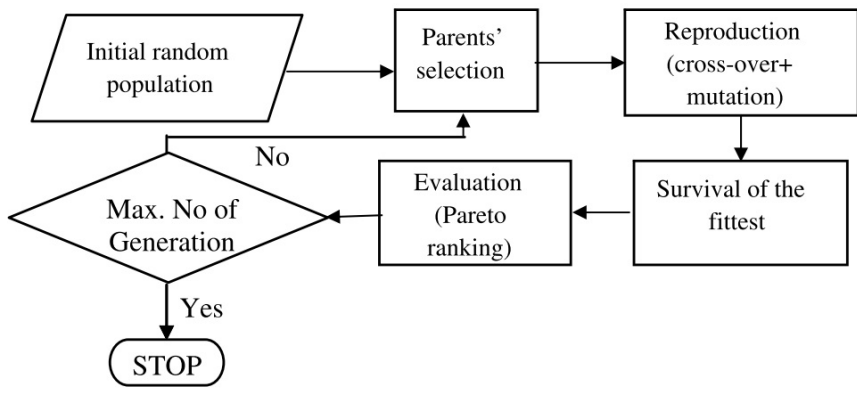

Fig. 2. Scheme for multi-objective evolutionary algorithm used in present study [35-37].

powerful optimization tool for nonlinear problems [28,44]. To create a hierarchy among the solutions, Pareto optimality is an important concept, helping to determine whether a solution is really one of the best possible tradesoffs [44]. In order to find the Pareto frontier, the NSGA-II algorithm is utilized as a multi-objective optimization method. Details and recent trends for evolutionary algorithms are reported elsewhere [28, 29,34].

\subsection{Objective functions, decision variables and constraints}

Three objective functions are used in this study: system power output $P_{o t}$, thermal efficiency $\eta_{o t}$ and entropy generation $\sigma_{o t}$, described by Equations (16), (17) and (23), respectively.

Also, three decision variables are considered: friction coefficient $\mu^{\prime}$, compression ratio $\gamma$, and a constant related to heat transfer $B$ (in $\mathrm{kJ} \cdot \mathrm{kg}^{-1} \cdot \mathrm{K}^{-1}$ ).

Although the decision variables may be varied in the optimization procedure, each is normally required to lie within a reasonable range. Hence, the objective functions are solved with respect to following constraints:

$$
\begin{gathered}
0.1 \leq \mu^{\prime} \leq 1.5 \\
0.01 \leq B \leq 0.03 \\
6 \leq \gamma \leq 10
\end{gathered}
$$

\section{Decision-making with multi-objective optimization}

A decision-making process for the selection of the final optimal answer from obtainable solutions is needed in multi-objective optimization. Various methods can be utilized to select a final optimum solution from the Pareto frontier. Before making a decision, the dimensions and scales of the objective space need to be unified, since the dimensions of various objectives in a multi-objective optimization issue may differ. Accordingly, objective vectors should be non-dimensioned before decision-making procedures are invoked. Euclidean and fuzzy non-dimensionalization methods can be used for non-dimensionalization. 


\subsection{Non-dimensionalization methods}

\subsubsection{Euclidean non-dimensionalization}

The matrix of objectives for various solutions of the Pareto frontier is denoted by $F_{i j}^{n}$ where $i$ is an index for each solution on the Pareto frontier and $j$ is an index for each objective in objective space. Then, a nondimensionalized objective, $F_{i j}^{n}$, can be defined as

$$
\begin{aligned}
& F_{i j}^{n}= \frac{F_{i j}}{\sqrt{\sum_{i=1}^{m}\left(F_{i j}\right)^{2}}} \\
& \text { (for minimizing and maximizing objectives) }
\end{aligned}
$$

\subsubsection{Fuzzy non-dimensionalization}

In this method, a non-dimensioned objective, $F_{i j}^{n}$, is defined as

$F_{i j}^{n}=\frac{F_{i j}-\min \left(F_{i j}\right)}{\max \left(F_{i j}\right)-\min \left(F_{i j}\right)} \quad$ (for maximizing objectives)

$F_{i j}^{n}=\frac{\max \left(F_{i j}\right)-F_{i j}}{\max \left(F_{i j}\right)-\min \left(F_{i j}\right)} \quad$ (for minimizing objectives)

In this work, the fuzzy Bellman-Zadeh, LINMAP and TOPSIS methods, which are well-known decision-making process types are provided in parallel, and the final optimal answer is selected by these three methods. The LINMAP and TOPSIS methods employ Euclidean nondimensionalization while the Bellman-Zadeh method employs fuzzy non-dimensionalization.

\subsection{Decision making methods}

\subsubsection{Bellman-Zadeh decision-making approach}

A final decision is defined by the Bellman and Zadeh approach as the intersection of all fuzzy criteria and limitations and is denoted by its membership function. The fuzzy membership function for each answer is set at the minimum value of the membership functions of all objectives for the suggested answer. In this method a maximum of minimums is chosen as an ultimate optimum answer. More explanations of the Bellman-Zadeh approach are demonstrated in reference [45].

\subsubsection{LINMAP decision-making approach}

An ideal point on the Pareto frontier is a point at which each objective is optimized regardless of optimizing the other objectives are fulfilled. In this approach, an answer with a minimum spatial distance from the ideal point is chosen as an ultimate optimum answer. More explanations of the LINMAP approach are demonstrated in reference [45].

\subsubsection{TOPSIS decision-making approach}

The non-ideal point is the ordinate in objective space in which each objective has its worst value. In this approach an answer with a maximum distance from the nonideal point and minimum distance from the ideal point is considered as an ultimate optimum answer. More explanations of the TOPSIS approach are demonstrated in reference [45].

\section{Results and discussion}

The output power and thermal efficiency of the Otto cycle are maximized and the entropy generation is minimized simultaneously using multi-objective optimization based on the NSGA-II algorithm. In this optimization, the objective functions are expressed by Equations (16), (17) and (23) and the constraints by Equations (25)-(27). The design parameters (decision variables) for the optimization follow: friction coefficient, a constant related to heat transfer and compression ratio.

Following reference [42], the following parameters are used here: $X_{1}=8 \times 10^{-2} \mathrm{~m}, X_{2}=1 \times 10^{-2} \mathrm{~m}$, $T_{1}=350 \mathrm{~K}, T_{3}=2200 \mathrm{~K}, T_{0}=300 \mathrm{~K}, n=30$, $C_{v}=0.7175 \mathrm{~kJ} \cdot \mathrm{kg}^{-1} \cdot \mathrm{K}^{-1}, M=4.553 \times 10^{-3} \mathrm{~kg} \cdot \mathrm{s}^{-1}$, $\eta_{e}=1$, and $\eta_{c}=1$.

The Pareto optimal frontier for the three objective functions (Otto cycle output power, thermal efficiency and system entropy generation) is presented in Figure 3. The chosen points based on various decision making methods are shown, too.

The Pareto optimal frontier for the two objective functions output power and thermal efficiency is shown in Figure 4. According to high value of $R^{2}$ resulted curve is accurate. It is clear that thermal efficiency is between 0.196 and 0.205 in the curve fitted diagram. That is,

$$
P_{o t}=A_{1}^{\prime} \eta_{o t}^{5}+A_{2}^{\prime} \eta_{o t}^{4}+A_{3}^{\prime} \eta_{o t}^{3}+A_{4}^{\prime} \eta_{o t}^{2}+A_{5}^{\prime} \eta_{o t}+A_{6}^{\prime}
$$

Coefficients:

$A_{1}^{\prime}=-0.0003978, \quad A_{2}^{\prime}=-0.00165, A_{3}^{\prime}=-0.002114$,

$A_{4}^{\prime}=-0.002408, A_{5}^{\prime}=-0.006069, A_{6}^{\prime}=1.832$.

Goodness of fit: $R^{2}=0.9994$.

The Pareto optimal frontier for the objective functions system entropy generation and thermal efficiency is shown in Figure 5. According to high value of $R^{2}$ resulted curve is accurate. The thermal efficiency is seen to be between 0.196 and 0.205 in the curve fitted diagram, i.e.,

$$
\sigma_{o t}=B_{1} \eta_{o t}^{4}+B_{2} \eta_{o t}^{3}+B_{3} \eta_{o t}^{2}+B_{4} \eta_{o t}+B_{5}
$$

Coefficients:

$$
\begin{aligned}
& B_{1}=-5.04 \times 10^{-6}, B_{2}=-1.866 \times 10^{-5}, \\
& B_{3}=-2.718 \times 10^{-5}, B_{4}=-0.0001242, B_{5}=0.002315 .
\end{aligned}
$$

Goodness of fit: $R^{2}=0.9997$. 


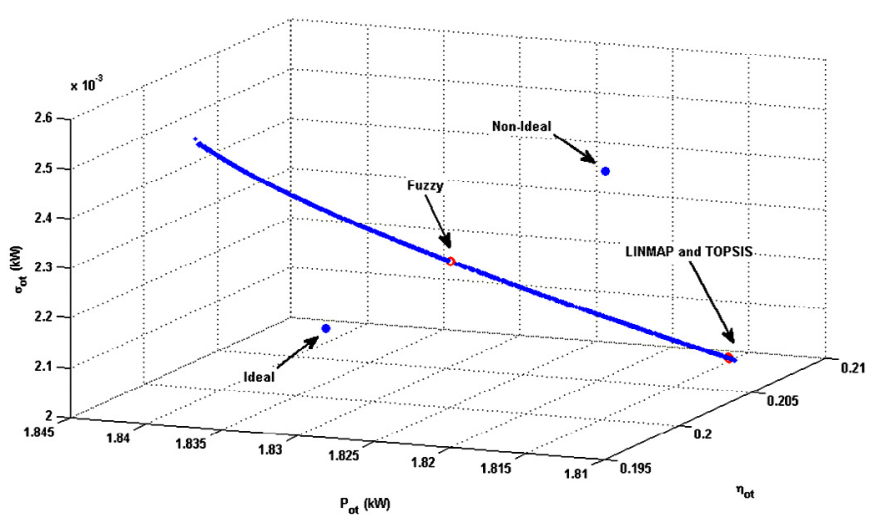

Fig. 3. Pareto optimal frontier in objective space.

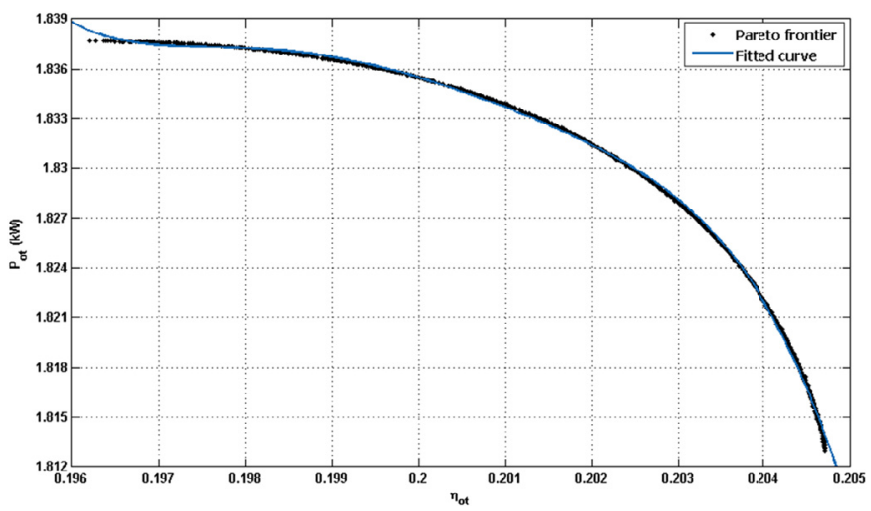

Fig. 4. Pareto optimal frontier in objective space $P_{o t}-\eta_{o t}$.

Finally, the Pareto optimal frontier for system entropy generation and output power is shown in Figure 6, where the output power is seen to lie between $1.81 \mathrm{~kW}$ and $1.84 \mathrm{~kW}$ in the curve fitted diagram:

$$
\sigma=C_{1} P^{5}+C_{2} P^{4}+C_{3} P^{3}+C_{4} P^{2}+C_{5} P+C_{6}
$$

Coefficients:

$$
\begin{aligned}
& C_{1}=9.841 \times 10^{-6}, C_{2}=2.878 \times 10^{-5}, C_{3}=1.398 \times 10^{-5}, \\
& C_{4}=1.137 \times 10^{-6}, C_{5}=0.0001079, C_{6}=0.002257 .
\end{aligned}
$$

Goodness of fit: $R^{2}=0.9986$.

The optimal results for decision parameters and objective functions using LINMAP, TOPSIS, and BellmanZadeh decision-making methods are summarized and compared in Table 1. Further, the maximum and mean error analyses for the objective functions are categorized in Table 2, where results of analyses for all mentioned decision-making methods are given. The results are promising and it is expected that the present study enhances understanding of the optimal design of the Otto cycle based on thermal criteria.

\section{Conclusions}

A thermodynamic optimization methodology has been applied to determine the output power, the thermal efficiency and the rate of entropy generation of the Otto

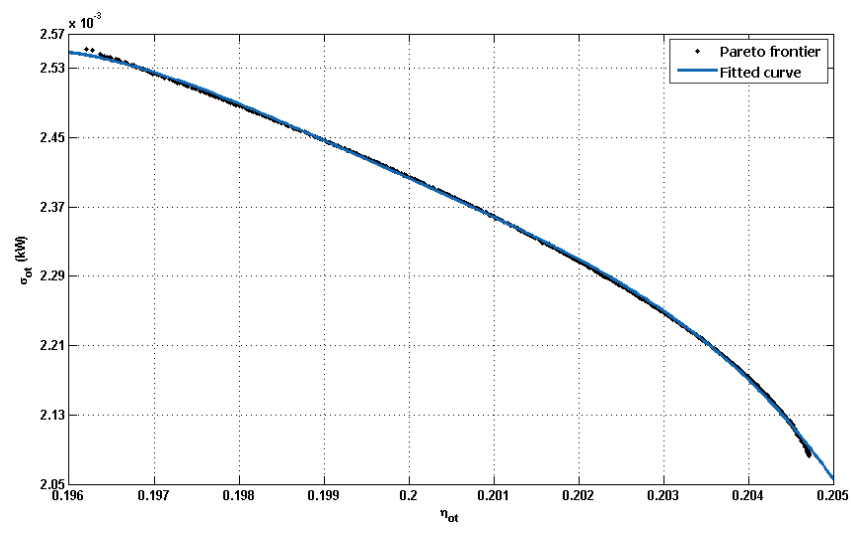

Fig. 5. Pareto optimal frontier in objective space $\sigma_{o t}-\eta_{o t}$.

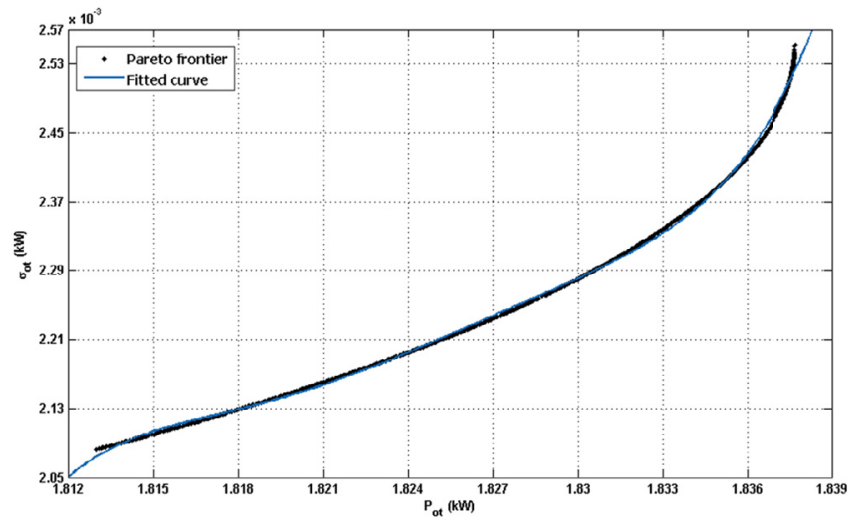

Fig. 6. Pareto optimal frontier in objective space $\sigma_{o t}-P_{o t}$.

Table 1. Decision making among multi-objective optimal solutions.

\begin{tabular}{ccccccc}
\hline \multirow{2}{*}{ Method } & \multicolumn{3}{c}{ Decision variables } & \multicolumn{3}{c}{ Objectives } \\
\cline { 2 - 7 } & $\mu$ & $B$ & $\gamma$ & $\eta_{\text {ot }}$ & $P_{\text {ot }}$ & $\sigma_{o t}$ \\
\hline Fuzzy & 0.100328 & 0.010024 & 9 & 0.203029 & 1.827829 & 0.002247 \\
LINMAP & 0.100134 & 0.010024 & 10 & 0.204827 & 1.811358 & 0.002068 \\
TOPSIS & 0.10005 & 0.010025 & 10 & 0.204832 & 1.811302 & 0.002068 \\
\hline
\end{tabular}

cycle. The effects of the friction coefficient, a constant related to heat transfer and compression ratio are examined utilizing the NSGA-II algorithm. The results can be implemented for designing and assessing Otto cycle power plant performance and robustness. A final optimal solution was selected from solutions in the Pareto frontier using three decision making methods (LINMAP, TOPSIS and fuzzy). An error analysis, performed based on the MAPE method, demonstrated that the average error of solutions obtained via the three decision making methods are $0.09 \%, 0.06 \%$ and $0.67 \%$ for the output power, the thermal efficiency and the entropy generation, respectively. The maximum errors of the solutions obtained by the three decision making methods are $0.14 \%, 0.12 \%$ and $1.01 \%$ for output power, thermal efficiency and entropy generation, respectively. 
M.H. Ahmadi et al.: Mechanics \& Industry 17, 111 (2016)

Table 2. Error analysis based on the mean absolute percent error (MAPE) method.

\begin{tabular}{cccccccccc}
\hline \multirow{2}{*}{ Error } & \multicolumn{3}{c}{ LINMAP } & \multicolumn{3}{c}{ TOPSIS } & \multicolumn{3}{c}{ Fuzzy } \\
\cline { 2 - 10 } & $\eta_{o t}$ & $P_{o t}$ & $\sigma_{o t}$ & $\eta_{o t}$ & $P_{o t}$ & $\sigma_{o t}$ & $\eta_{o t}$ & $P_{o t}$ & $\sigma_{o t}$ \\
\hline Max. error (\%) & 0.12 & 0.05 & 0.59 & 0.07 & 0.14 & 1.00 & 0.07 & 0.13 & 1.01 \\
Average error (\%) & 0.06 & 0.04 & 0.45 & 0.03 & 0.09 & 0.67 & 0.03 & 0.09 & 0.65 \\
\hline
\end{tabular}

\section{References}

[1] B. Andresen, R.S. Berry, M.J. Ondrechen, P. Salamon, Thermodynamics for processes in finite time, Acc. Chem. Res. 17 (1984) 266-271

[2] A. Bejan, Entropy generation on minimization: The new thermodynamics of finite-size device and finite-time processes, J. Appl. Phys. 79 (1996) 1191-1218

[3] M. Feidt, Thermodynamique et Optimisation Energétique des Systèmes et Procédés, Technique et Documentation, 2nd edn., Lavoisier, Paris, 1996

[4] R.S. Berry, V.A. Kazakov, S. Sieniutycz, Z. Szwast, A.M. Tsirlin, Thermodynamic Optimization of Finite Time Processes, Wiley, Chichester, 1999

[5] L. Chen, C. Wu, F. Sun, Finite time thermodynamic optimization or entropy generation minimization of energy systems, J. Non-Equilib. Thermodyn. 24 (1999) 327-359

[6] L. Chen, F. Sun, Advances in Finite Time Thermodynamics: Analysis and optimization, Nova Science Publishers, New York, 2004

[7] M. Feidt, Optimal use of energy systems and processes, Int. J. Exergy 5 (2008) 500-531

[8] S. Sieniutycz, J. Jezowski, Energy Optimization in Process Systems, Elsevier, Oxford, 2009

[9] M. Feidt, Thermodynamics applied to reverse cycle machines: a review, Int. J. Refrig. 33 (2010) 1327-1342

[10] B. Andresen, Current trends in finite-time thermodynamics, Angew. Chem. Int. Edit. 50 (2011) 2690-2704

[11] S.A. Klein, An explanation for observed compression ratios in internal combustion engines, Trans. ASME J. Eng. Gas Turbine Power 113 (1991) 511-513

[12] C. Wu, D.A. Blank, The effect combustion on a workoptimized endoreversible Otto cycle, J. Energy Inst. 65 (1992) 86-89

[13] D.A. Blank, C. Wu, Optimization of the endoreversible Otto cycle with respect to both power and mean effective pressure, Energy Convers. Manage. 34 (1993) 1255-1209

[14] L. Chen, C. Wu, F. Sun, Heat transfer effects on the network output and efficiency characteristics for an air standard Otto cycle, Energy Convers. Manage. 39 (1998) 643-648

[15] A. Ficher, K.H. Hoffman, Can a quantitative simulation of an Otto engine be accurately rendered by a simple Novikov model with heat leak, J. Non-Equilib. Thermodyn. 29 (2004) 9-28

[16] O.A. Ozsoysal, Heat loss as a percentage of fuel's energy in air standard Otto and Diesel cycles, Energy Convers. Manage. 47 (2006) 1051-1062

[17] S.S. Hou, Comparison of performances of air standard Atkinson and Otto cycles with heat transfer considerations, Energy Convers. Manage. 48 (2007) 1683-1690

[18] F. Angulo-Brown, J. Fernandez-Betanzos, C.A. DiazPico, Compression ratio of an optimized Otto-cycle model, Eur. J. Phys. 15 (1994) 38-42
[19] L. Chen, T. Zheng, F. Sun, C. Wu, The power and efficiency characteristics for an irreversible Otto cycle, Int. J. Ambient Energy 24 (2003) 195-200

[20] F. Angulo-Brown, J.A. Rocha-Martinez, T.D. NavarreteGonzalez, A non-endoreversible Otto cycle model: improving power output and efficiency, J. Phys. D 29 (1996) $80-83$

[21] J. Chen, Y. Zhao, J. He, Optimization criteria for the important parameters of an irreversible Otto heat-engine, Appl. Energy 83 (2006) 228-238

[22] Y. Zhao, J. Chen, Irreversible Otto heat engine with friction and heat leak losses and its parametric optimum criteria, J. Energy Inst. 81 (2008) 54-58

[23] M. Feidt, Optimal thermodynamics - new upper bounds, Entropy 11 (2009) 529-547

[24] J.A. Rocha-Matinez, T.D. Navarrete-Gonzalez, C.G. Pavia-Miller, et al., A simplified irreversible Otto-engine model with fluctuations in the combustion heat, Int. J. Ambient Energy 27 (2006) 181-92

[25] T. Özyer, M. Zhang, R. Alhajj, Integrating multiobjective genetic algorithm based clustering and data partitioning for skyline computation, Appl. Intell. 35 (2011) $110-122$

[26] O. Beatrice, J.R. Brian, H. Franklin, Multi-Objective Genetic Algorithms for Vehicle Routing Problem with Time Windows, Appl. Intell. 24 (2006) 17-30

[27] I. Blecic, A. Cecchini, G. Trunfio, A decision support tool coupling a causal model and a multi-objective genetic algorithm, Appl. Intell. 26 (2007) 125-137

[28] D.A.V. Veldhuizen, G.B. Lamont, Multiobjective Evolutionary Algorithms Analyzing the State-of-theArt, Evol. Comput. 8 (2000) 125-147

[29] A. Konak, D.W. Coit, A.E. Smith, Multi-objective optimization using genetic algorithms: A tutorial, Reliab. Eng. Syst. Safety 91 (2006) 992-1007

[30] S. Toghyani, A. Kasaeian, M.H. Ahmadi, Multi-objective optimization of Stirling engine using non-ideal adiabatic method, Energy Convers. Manage. 80 (2014) 54-62

[31] M.H. Ahmadi, H. Sayyaadi, A.H. Mohammadi, M.A. Barranco-Jimenez, Thermo-economic Multi-objective optimization of solar dish-Stirling engine by implementing evolutionary algorithm, Energy Convers. Manage. 73 (2013) 370-380

[32] S.A. Sadatsakkak, M.H. Ahmadi, M.A. Ahmadi, Optimization performance and thermodynamic analysis of an irreversible nano scale Brayton cycle operating with Maxwell-Boltzmann gas, Energy Convers. Manage. 101 (2015) 592-605

[33] M.H. Ahmadi, M.A. Ahmadi, Thermodynamic analysis and optimization of an irreversible Ericsson cryogenic refrigerator cycle, Energy Convers. Manage. 89 (2015) 147155 
[34] M.H. Ahmadi, M.A. Ahmadi, A.H. Mohammadi, M. Mehrpooya, M. Feidt, Thermodynamic optimization of Stirling heat pump based on multiple criteria, Energy Convers. Manage. 80 (2014) 319-328

[35] M.H. Ahmadi, M.A. Ahmadi, M. Mehrpooya, M. Sameti, Thermo-ecological analysis and optimization performance of an irreversible three-heat-source absorption heat pump, Energy Conversion and Management 90 (2015) $175-183$

[36] S.A. Sadatsakkak, M.H. Ahmadi, M.A. Ahmadi, Thermodynamic and thermo-economic analysis and optimization of an irreversible regenerative closed Brayton cycle.Energy Conversion and Management, 94 (2015) 124-129

[37] S.A. Sadatsakkak, M.H. Ahmadi, R. Bayat, S.M. Pourkiaei, M. Feidt, Optimization density power and thermal efficiency of an endoreversible Braysson cycle by using non-dominated sorting genetic algorithm. Energy Conversion and Management 93 (2015) 31-39

[38] Y. Ge, L. Chen, F. Sun, Ecological Optimization of an Irreversible Otto Cycle, Arab J. Sci. Eng. 38 (2013) 373381

[39] Y. Ge, L. Chen, F. Sun, Finite time thermodynamic modeling and analysis for an irreversible Otto cycle, Appl. Energy 85 (2008) 618-624
[40] M. Mozurkewich, R.S. Berry, Finite-time thermodynamics: engine performance improved by optimized piston motion, Proc. Natl. Acad. Sci. USA 78 (1981) 1986- 1988

[41] M. Mozurkewich, R.S. Berry, Optimal paths for thermodynamic systems: the ideal Otto cycle, J. Appl. Phys. 53 (1982) 34-42

[42] L. Chen, Y. Ge, F. Sun, C. Wu, Effects of heat transfer friction and variable specific heats of working fluid on performance of an irreversible Dual cycle, Energy Convers. Manage. 47 (2006) 3224-3234

[43] L. Chen, J. Zhou, F. Sun, C. Wu, Ecological optimization for generalized irreversible Carnot engines, Appl. Energy 77 (2004) 327-338

[44] T.D. Back, Z. Fogel Michalewicz, Handbook of evolutionary computation, Oxford Univ. Press, 1997

[45] M.H. Ahmadi, H. Hosseinzade, H. Sayyaadi, A.H. Mohammadi, F. Kimaghalam, Application of the multiobjective optimization method for designing a powered Stirling heat engine: Design with maximized power, thermal efficiency and minimized pressure loss, Renew. Energy 60 (2013) 313-322 